\title{
Cytological characteristics of meningeal solitary fibrous tumor metastatic to the lung: A case report with immunocytochemical analysis
}

\author{
KAORI SANDOH $^{1 *}$, MITSUAKI ISHIDA $^{1 *}$, KIMIAKI OKANO $^{1}$, YUSUKE EBISU ${ }^{1}$, \\ KENTO FUKUMOTO $^{2}$, TOMOHITO SAITO ${ }^{2}$, TOMOHIRO MURAKAWA ${ }^{2}$, TOKIKO NAKAI ${ }^{3}$, \\ KINTA HATAKEYAMA ${ }^{3}$, CHIHO OHBAYASHI ${ }^{3}$ and KOJI TSUTA ${ }^{1}$
}

\begin{abstract}
Departments of ${ }^{1}$ Pathology and Laboratory Medicine, and ${ }^{2}$ Thoracic Surgery, Kansai Medical University, Hirakata, Osaka 573-1010; ${ }^{3}$ Department of Diagnostic Pathology, Nara Medical University, Kashihara, Nara 634-0813, Japan
\end{abstract}

Received March 22, 2018; Accepted May 11, 2018

DOI: $10.3892 / \mathrm{mco} .2018 .1625$

\begin{abstract}
Solitary fibrous tumor (SFT) is a relatively uncommon mesenchymal tumor, and its occurrence in the meninges is rare. We herein report what is, to the best of our knowledge, the first cytological case of meningeal SFT metastatic to the lung with immunocytochemical analysis for signal transducer and activator of transcription 6 (STAT6), and compare the cytological characteristics to those of pleuropulmonary SFT. A 58-year-old Japanese male patient was found to have multiple nodules in the bilateral lungs after surgery for meningeal SFT. Partial resection of the nodules was performed, and touch smears were obtained. The Papanicolaou smear revealed cohesive hypercellular clusters of polygonal to elongated neoplastic cells with scant cytoplasm and oval to short spindle-shaped nuclei with nucleoli containing coarse chromatin. Mild-to-moderate nuclear pleomorphism was observed. No collagenous stroma was noted. Immunocytochemical analysis revealed that the neoplastic cells diffusely expressed STAT6. Histopathological and immunohistochemical studies confirmed the diagnosis of meningeal SFT metastatic to the lung. Moreover, reverse transcription-polymerase chain reaction analysis revealed that the lung tumor harbored NAB2ex6-STAT6ex16 fusion. Recent studies demonstrated that there is a clinicopathological difference among NAB2-STAT6 fusion variants in SFT. As reflected in fusion variants, meningeal SFT occasionally lacks collagenous stroma, as in the present case, although the most characteristic cytological feature of SFT is the presence of spindle-shaped
\end{abstract}

Correspondence to: Dr Mitsuaki Ishida, Department of Pathology and Laboratory Medicine, Kansai Medical University, 2-5-1 Shinmachi, Hirakata, Osaka 573-1010, Japan

E-mail: ishidamt@hirakata.kmu.ac.jp

*Contributed equally

Key words: solitary fibrous tumor, meninges, lung metastasis, signal transducer and activator of transcription 6 neoplastic cells embedded in dense collagenous stroma. Thus, immunocytochemical analysis for STAT6 is very useful for diagnosing SFT.

\section{Introduction}

Solitary fibrous tumor (SFT) is a relatively rare mesenchymal tumor. SFT and hemangiopericytoma (HPC) were previously considered to be distinct disease entities; however, the discovery of NAB2-STAT6 gene fusion in the majority of SFT and HPC cases resulted in the recognition of these two entities as a single clinicopathological disease entity referred to as SFT $(1,2)$. SFT can affect virtually any site in the body; however, it occurs preferentially in the pleural cavity. Meningeal SFT is rare, accounting for $<1 \%$ of all central nervous system tumors (3). This type of tumor occasionally develops extracranial metastases, such as to the lung and liver (3). To the best of our knowledge, this is the first cytological report of primary meningeal SFT metastatic to the lung with an immunocytochemical analysis of signal transducer and activator of transcription 6 (STAT6), with a comparison of the cytological characteristics to those of pleuropulmonary SFT.

\section{Case report}

On February 2017, a 58-year-old Japanese male patient was found to have multiple nodules in the bilateral lungs by thoracic computed tomography at Kansai Medical University Hospital 6 years following surgery for right meningeal SFT. Partial resection of the right lung nodules (2 lesions from the upper lobe, and 1 lesion each from the middle and lower lobes) was performed following a clinical diagnosis of metastatic SFT. Touch smears of the lung nodules were performed. Specimens of the tumor touch smears were stained with Papanicolaou stain.

Formalin-fixed and paraffin-embedded specimens of the resected tumors were processed for routine histological examination and immunohistochemical analyses.

In thisreport,immunohistochemical and immunocytochemical analyses were performed using an autostainer (XT System 
A

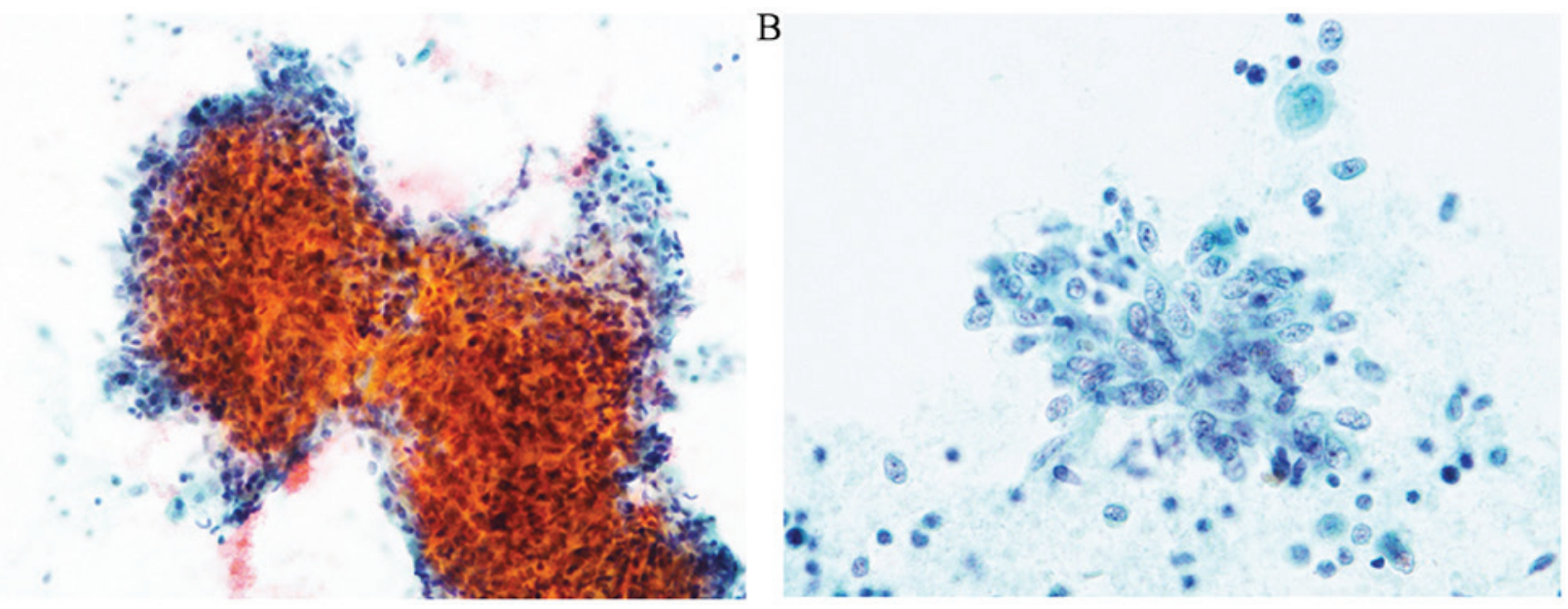

Figure 1. Cytological findings of the touch smear of the lung nodule. (A) Cohesive hypercellular cluster of polygonal to elongated neoplastic cells without collagenous stroma and dilated vessel structure (Papanicolaou stain; magnification, x100). (B) The neoplastic cells had oval to short spindle-shaped nuclei and scant cytoplasm. Mild-to-moderate nuclear pleomorphism was observed (Papanicolaou stain; magnification, x400).

Benchmark, Roche Diagnostics, Basel, Switzerland, and Autostainer link 48; DAKO Cytomation, Glostrup, Denmark). The primary antibodies used in this report were mouse monoclonal antibody against B-cell lymphoma 2 (Bcl-2; 124), mouse monoclonal antibody against CD34 (QBEnd10), mouse monoclonal antibody against Ki-67 (MIB1) (all from DAKO) and mouse monoclonal antibody against STAT6 (D-1, Santa Cruz Biotechnology, Dallas, TX, USA).

For detection of NAB2-STAT6 fusion, reverse transcription-polymerase chain reaction (RT-PCR) analysis was performed.

Cytological findings of the tumor touch smears. The Papanicolaou smear revealed cohesive hypercellular clusters or sheets of polygonal to elongated neoplastic cells in an inflammatory background (Fig. 1A). These neoplastic cells had scant cytoplasm and oval to short spindle-shaped nuclei with small nucleoli containing coarse chromatin (Fig. 1B). Mild-to-moderate nuclear pleomorphism was observed (Fig. 1B). Neither collagenous stroma nor dilated vascular structures were noted. Mitotic figures were not found.

Immunocytochemical findings. STAT6 was diffusely expressed in the nuclei of the neoplastic cells (Fig. 2). Accordingly, metastatic SFT was suspected.

Histopathological findings. The four resected pulmonary lesions exhibited fundamentally the same characteristics. The tumors were composed of sheet-like proliferations of polygonal to short spindle-shaped neoplastic cells (Fig. 3A). These neoplastic cells had scant cytoplasm and oval to short spindle-shaped nuclei with small nucleoli containing coarse chromatin (Fig. 3B). Slit-like vessels were focally observed; however, fibrous stroma was absent.

A review of the slides revealed that the histopathological characteristics of the meningeal tumor were identical to those of the lung lesions.

Immunohistochemical findings. The neoplastic cells in the lung diffusely expressed STAT6, Bcl-2 and CD34 (Fig. 4). The

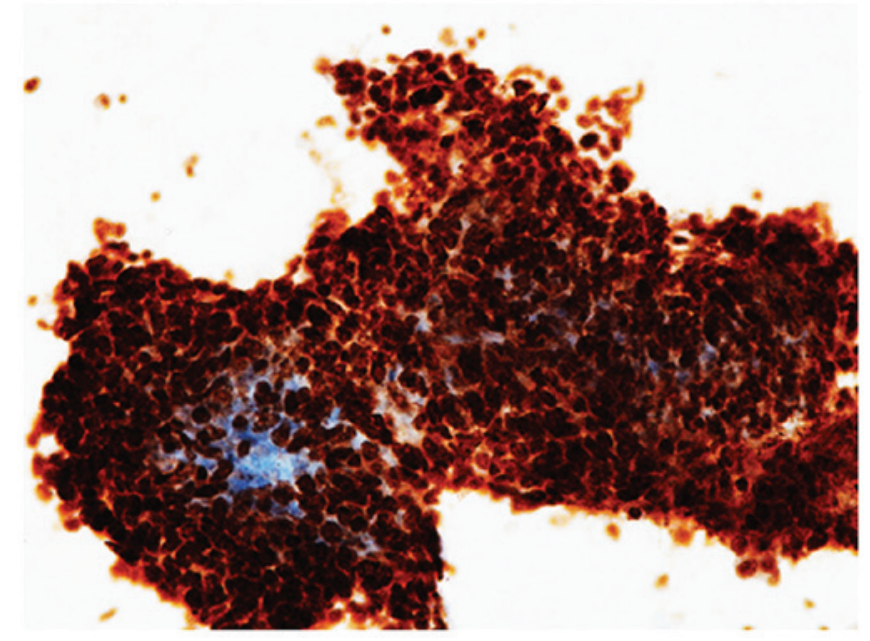

Figure 2. Immunocytochemical findings. STAT6 is diffusely expressed (magnification, x400). STAT6, signal transducer and activator of transcription 6.

meningeal tumor displayed the same immunohistochemical characteristics, and the Ki-67 labeling index was $1 \%$. Accordingly, the diagnosis of meningeal SFT metastatic to the lung was confirmed.

RT-PCR. The lung tumor exhibited NAB2ex6-STAT6ex16 fusion.

\section{Discussion}

In this article, we described the first cytological report of meningeal SFT metastatic to the lung. The characteristic cytological features of extracranial SFT are as follows: i) Presence of hypercellular clusters, ii) oval, elongated, round, or stellate neoplastic cells with limited nuclear pleomorphism, iii) neoplastic cells with wispy cytoplasm or naked nuclei and iv) presence of pink collagenous intercellular stroma and occasional vessel-like structures (4). In particular, the presence of collagenous intercellular stroma is characteristic for this type of tumor, and this finding is observed in almost all cases of benign SFTs (99.5\%) (4). Moreover, it has been reported that cytological characteristics indicative of malignant SFT are 

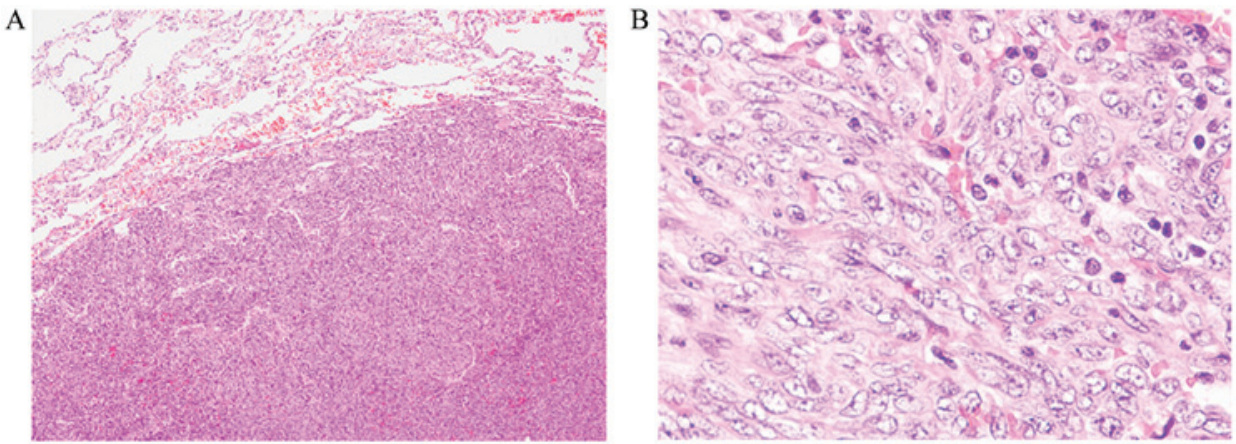

Figure 3. Histopathological characteristics of the lung nodule. (A) Sheet-like proliferation of polygonal to short spindle-shaped neoplastic cells without fibrous stroma and dilated vessel structure [hematoxylin and eosin (H\&E) stain; magnification, x100]. (B) The neoplastic cells had oval to short spindle-shaped nuclei and scant cytoplasm (H\&E stain; magnification, $\mathrm{x} 400)$.
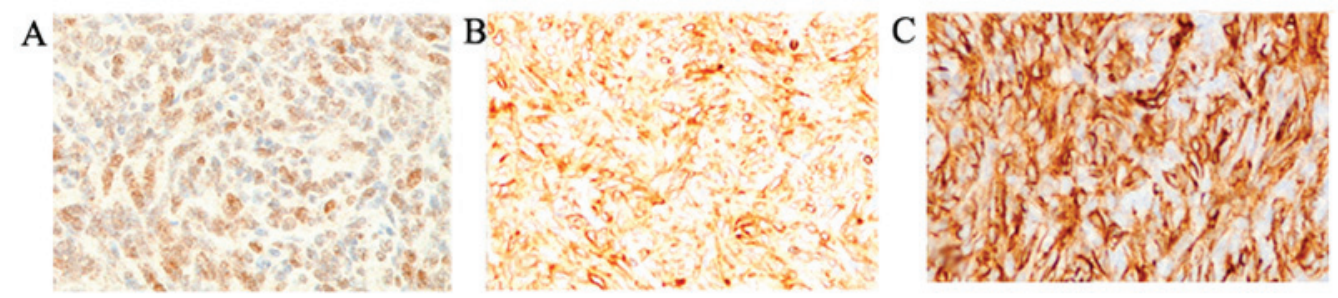

Figure 4. Immunohistochemical characteristics of the lung nodule. (A) STAT6, (B) Bcl-2 and (C) CD34 are diffusely expressed (magnification, x400). STAT6, signal transducer and activator of transcription 6; Bcl-2, B-cell lymphoma 2.

the presence of moderate to extensive nuclear pleomorphism, mitotic figures and necrosis (4). In the present case, the presence of hypercellular clusters composed of polygonal to elongated neoplastic cells with scant cytoplasm and oval to short spindle-shaped nuclei corresponded to the above mentioned cytological characteristics. The striking cytological finding in the present case was the absence of collagenous stroma, which is noted in $14 \%$ of malignant SFTs, and this makes it difficult to diagnose SFT. Thus, immunocytochemical analysis for STAT6 is required to confirm the diagnosis of SFT (described below).

SFT displays a spectrum of histopathological characteristics. Conventional (typical) SFT exhibits proliferation of spindle cells with scant cytoplasm and uniform oval to spindled nuclei within a prominent fibrous stroma and staghorn vessels. This paucicellular type is classified as fibrous SFT. Tumors with higher cellularity and more rounded cells, but with prominent perivascular fibrosis, are referred to as cellular SFT, whereas highly cellular tumors with well-developed thin-walled or dilated vessels without perivascular fibrosis are classified as HPC (5). Interestingly, Barthelmess et al reported that there is a clinicopathological difference among NAB2-STAT6 fusion variants (5). NAB2ex4-STAT6ex $2 / 3$ corresponds to the classical thoracic SFT with fibrous histology and better prognosis. By contrast, NAB2ex6-STAT6ex16/17 represents the cellular SFT and HPC histology, with more aggressive behavior and extrathoracic occurrence (5). Moreover, it has been documented that over half of the cases of meningeal SFT harbor NAB2ex6-STAT6ex16/17 fusion, and the histopathological characteristics also reflect the fusion variant in the meningeal SFT, as well as non-meningeal SFT (6). In the present case, the metastatic tumor harbored NAB2ex6-STAT6ex16 fusion, consistently with the histopathological and cytological characteristics of HPC-type SFT.

STAT6 has been recognized as a useful immunohistochemical marker for both meningeal and non-meningeal SFTs, without regard for fusion variants (5-8). Moreover, the usefulness of the immunocytochemical analysis for STAT6 in formalin-fixed fine-needle aspiration specimens has also been reported (4). In the present case, immunocytochemical staining for STAT6 led to cytodiagnosis of metastatic SFT, although the cytological characteristics were not typical of conventional SFT, including the absence of collagenous stroma. Therefore, immunocytochemical analysis for STAT6 is recommended when SFT is suspected clinically and cytologically.

\section{Acknowledgements}

Not applicable.

\section{Funding}

No funding was received.

\section{Availability of data and materials}

Not applicable.

\section{Authors' contributions}

KS, MI and KO contributed to cytological diagnosis and manuscript preparation. YE performed immunocytochemical and immunohistochemical staining. MI and KT performed histopathological diagnosis. KF, TS and TM contributed to 
patient data collection. TN, KH and CO performed RT-PCR. The final version of the manuscript has been read and approved by all authors.

\section{Ethics approval and consent to participate}

Not applicable.

\section{Consent for publication}

The authors obtained the consent of the patient to the publication of the case details.

\section{Competing interests}

The authors declare that they have no competing interests.

\section{References}

1. Chmielecki J, Crago AM, Rosenberg M, O'Connor R, Walker SR, Ambrogio L, Auclair D, McKenna A, Heinrich MC, Frank DA and Meyerson M: Whole-exome sequencing identifies a recurrent NAB2-STAT6 fusion in solitary fibrous tumors. Nat Genet 45 : 131-132, 2013.

2. Robinson DR, Wu YM, Kalyana-Sundaram S, Cao X, Lonigro RJ, Sung YS, Chen CL, Zhang L, Wang R, Su F, et al: Identification of recurrent NAB2-STAT6 gene fusions in solitary fibrous tumor by integrative sequencing. Nat Genet 45: 180-185, 2013
3. Giannini C, Rushing EJ, Hainfellner JA, Bouvier C, Figarella-Branger D, von Deimling A, Wesseling $P$ and Antonescu CR: Solitary fibrous tumour/haemangiopericytoma. In: WHO Classification of Tumours of the Central Nervous System. Revised 4th edition. Louis D, Ohgaki H, Wiestler OD and Cavenee WK (eds). IARC, Lyon, pp249-254, 2016.

4. Tani E, Wejde J, Åström K, Wingmo IL, Larsson O and Haglund F: FNA cytology of solitary fibrous tumors and the diagnostic value of STAT6 immunocytochemistry. Cancer Cytopathol 126: 36-43, 2018.

5. Barthelmess S, Geddert H, Boltze C, Moskalev EA, Bieg M, Sirbu H, Brors B, Wiemann S, Hartmann A, Agaimy A and Haller F: Solitary fibrous tumors/hemangiopericytomas with different variants of the NAB2-STAT6 gene fusion are characterized by specific histomorphology and distinct clinicopathological features. Am J Pathol 184: 1209-1218, 2014.

6. Yuzawa S, Nishihara H, Wang L, Tsuda M, Kimura T, Tanino M and Tanaka S: Analysis of NAB2-STAT6 gene fusion in 17 cases of meningeal solitary fibrous tumor/hemangiopericytoma: Review of the literature. Am J Surg Pathol 40: 1031-1040, 2016.

7. Schweizer L, Koelsche C, Sahm F, Piro RM, Capper D, Reuss DE, Pusch S, Habel A, Meyer J, Göck T, et al: Meningeal hemangiopericytoma and solitary fibrous tumors carry the NAB2-STAT6 fusion and can be diagnosed by nuclear expression of STAT6 protein. Acta Neuropathol 125: 651-658, 2013.

8. Koelsche C, Schweizer L, Renner M, Warth A, Jones DT, Sahm F, Reuss DE, Capper D, Knösel T, Schulz B, et al: Nuclear relocation of STAT6 reliably predicts NAB2-STAT6 fusion for the diagnosis of solitary fibrous tumour. Histopathology 65: 613-622, 2014 International (CC BY-NC-ND 4.0) License. 